\title{
Genetic specification of life span and self-fertility in recombinant-inbred strains of Caenorhabditis elegans
}

\author{
ANNE BROOKS \& THOMAS E. JOHNSON \\ Institute for Behavioral Genetics and Department of Psychology, Campus Box 447, University of Colorado, Boulder, \\ Colorado 80309, USA
}

\begin{abstract}
The genetic basis of life-span and age-specific fertility has been analysed using recombinant-inbred strains of the nematode Caenorhabditis elegans. Estimates of narrow-sense heritability range from 0.05 to 0.36 for life span and from 0.36 to 0.49 for total self-fertility. Positive phenotypic and genetic correlations for life span and total fertility were also observed, although in most cases the correlations were not significant. In general, age-specific hermaphrodite fertility was positively correlated with fertility on contiguous days but was negatively correlated with fertility on more distant days. We estimate that a minimum of two to three genes specify each of these traits in this genetic background. Three single-gene markers were used to generate strain distribution patterns, and two of these were found to be linked with loci that specify life span and/or fertility. We also saw evidence for a significant environmental component affecting self-fertility.
\end{abstract}

Keywords: antagonistic pleiotropy, fertility, life-history, life span, recombinant inbred, selective breeding.

\section{Introduction}

Antagonistic pleiotropy is one mechanism offered by evolutionary biologists to explain senescence. The theory of antagonistic pleiotropy states that genes with a beneficial effect early in life will be favoured even if they are deleterious to the organism later in life because the force of natural selection diminishes after the reproductive phase is complete. Williams (1957) proposed that senescence evolved because alleles that increase reproductive success during early adulthood are favoured, even though such genes may pleiotropically decrease survival in late adulthood. We report genetic tests of this theory in the nematode Caenorhabditis elegans using recombinant-inbred (RI) strains.

RI strains are produced by crossing genetically divergent parental strains followed by inbreeding of the hybrid progeny into a number of distinct strains (Bailey, 1971). Differences among RI strains result from unique combinations of parental chromosome regions found within each RI strain. C. elegans is particularly suited to RI research for three reasons. First, the unusual mating system of $C$. elegans [i.e. selffertilizing hemaphrodites that can be outcrossed to males (Nigon, 1949; Brenner 1974)] permits rapid inbreeding. Second, the short life cycle of 3-4 days permits approximately two generations per week. Third, the lack of inbreeding depression for life expectancy and fertility (Johnson \& Wood, 1982; T. E. Johnson, in preparation) in C. elegans simplifies the interpretation of the quantitative genetic analyses that follow. The $98 \%$ homozygosity that defines a RI strain (Bailey, 1971) can be achieved in six generations of selfing.

In this study we have used 16 strains of $C$. elegans (two parental and $14 \mathrm{RI}$ ) to estimate the relative magnitude of the genetic and environmental determinants of life span and self-fertility, to estimate the number of genes that specify these traits, and to examine the genetic and phenotypic correlations among life-span age-specific self-fertilities. We have also started genetic mapping of quantitative trait loci (QTLs; Lander \& Botstein, 1989) that specify these differences.

\section{Materials and methods}

\section{Media and rearing conditions}

Standard monoxenic techniques were used to rear $C$. elegans cultures on agar nutrient growth medium 
(NGM) with E. coli strain OP50 as the food source; these and other well established techniques are described elsewhere (Brenner, 1974; Wood 1988). Nematodes were kept at $20^{\circ} \mathrm{C}$ unless noted otherwise. The same 16 strains were measured on three different occasions and the conditions are summarized below. Trial 1 was started in March, 1983. Worms were transferred every $24 \mathrm{~h}$ during the fertile period. Individual cultures were started with 10 worms per strain. Trial 2 was started in May, 1986. Worms were transferred every $24 \mathrm{~h}$ during the fertile period. Twelve worms per strain were started for individual cultures. Trial 3 was started in December, 1986. Worms were transferred at 12-h intervals during their fertile period. The incubator temperature fluctuated during this time and rose as high as $23^{\circ} \mathrm{C}$.

\section{Nematode strains}

Sixteen $C$. elegans strains were used: two wild-type parental strains, Bristol (N2) and Bergerac Boulder (BergBo), plus 14 RI strains (Johnson \& Wood, 1982). These RI strains were generated from crosses between BergBO and N2, followed by 19 generations of selffertilization. The RI strains used were TJ101, TJ104, TJ106, TJ107, TJ119, TJ124, TJ128, TJ130, TJ132, TJ135, TJ138, TJ141, TJ142, and TJ143.

Both N2 and BergBO were obtained from David Hirsh. N2 is the Bristol strain (Brenner, 1974) originally obtained as an axenic culture from E. C. Dougherty (Nicholas et al., 1959). BergBO (sometimes referred to previously as Bergerac Lyon) was isolated by Nigon near Bergerac, France (Nigon, 1949) and was obtained by the Hirsh laboratory before the Bergerac strain was acquired by the Caenorhabditis Genetics Center for distribution. Some divergence of the Bergerac strain used in these analyses from the more commonly used Bergerac strain is apparent when Tc1 elements are characterized (C. Link and T. E. Johnson, unpublished observations)

Strains were maintained as dauer larvae at $16^{\circ} \mathrm{C}$ on starved, 'master' plates or were recovered from liquid nitrogen. This procedure avoids the accumulation of unwanted mutations that occurs during continuous subculture in the laboratory. To obtain experimental subjects either a plug of agar containing several nematodes was removed from each master or the strain was thawed onto a fresh plate of NGM seeded with $E$. coli. Progeny were isolated as fertile eggs by the hypochlorite method (Emmons et al., 1979). Eggs were allowed to hatch in sterile S-basal and first stage larvae (L1) were kept for up to 3 days until all strains were harvested by placing L1s on fresh NGM plates. Individual worms were transferred to seperate microtitre wells $48 \mathrm{~h}$ later when the nematodes were young adults. All temperatures were $20^{\circ} \mathrm{C}$ or room temperature during manipulations.

\section{Survival and fertility assays}

The standard procedures for survival assay (Johnson \& Wood, 1982; Friedman \& Johnson, 1988) were slightly

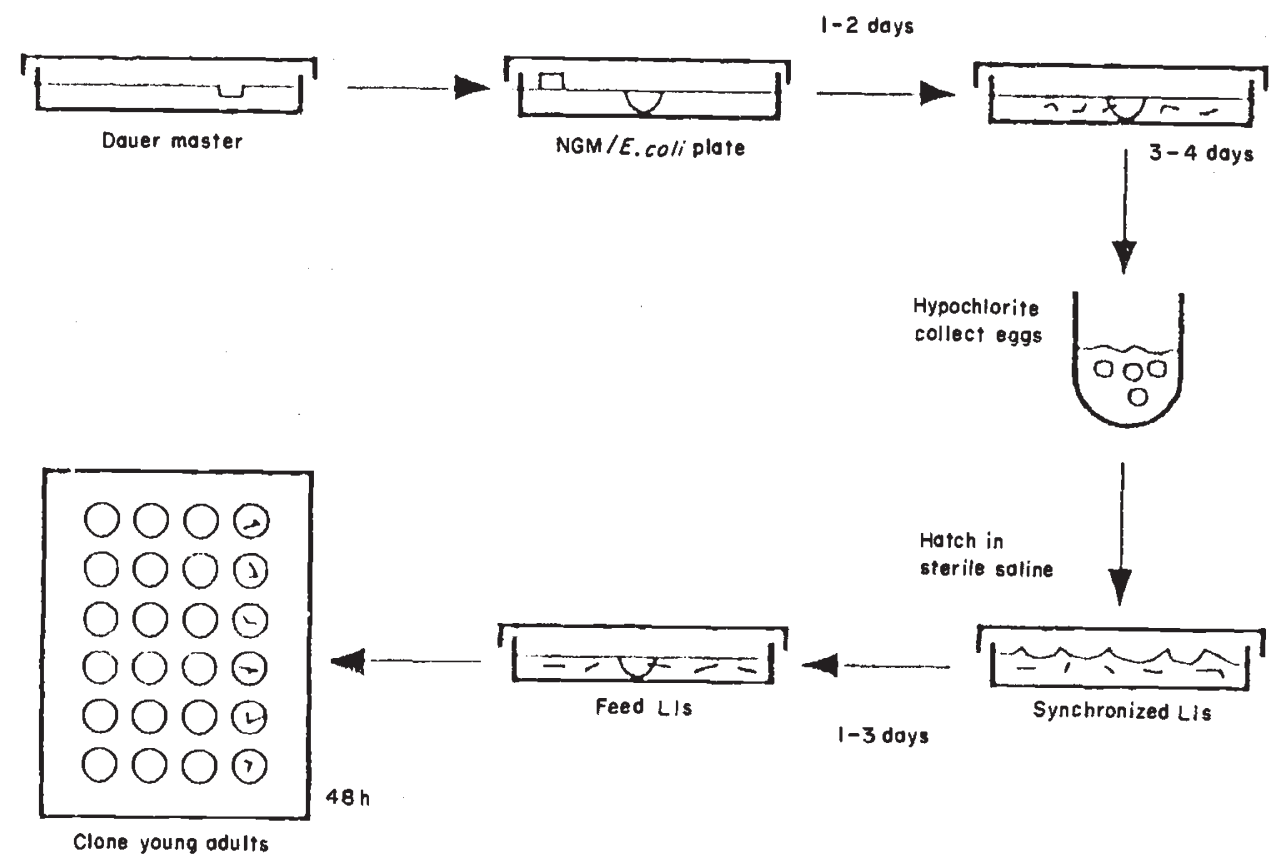

Fig. 1 Preparation of cultures that allow the measurements of individual survival and fertility. 
modified to allow assay of individual worms (Fig. 1). The time of death was taken as the date that the worm was scored as dead. The causes of death, other than old age (intrauterine hatchings, killed by experimenter, or lost), are not included in these survival results. Hermaphrodites were assayed for survival and transferred to fresh media daily for 10 days. After the fertile period, nematodes were assayed and transferred three times a week until few worms remained alive, when they were assayed three times a week and transferred once a week.

Daily progeny production by individual hermaphrodites was determined by counting offspring in each microtitre well when the progeny reached the fourth larval or young adult stage. The self-fertility was calculated only for those individuals who died from natural causes.

\section{Statistical tests}

All statistical test were performed using SYSTAT: The System for Statistics (Wilkinson, 1988). Phenotypic correlations were estimated using observations on individual worms; genetic correlations were estimated using means of the 16 different strains (Blizard \& Bailey, 1979). Survival comparisons were made using SURVIVAL: A Supplementary Module for SYSTAT (Steinberg \& Colla, 1988).

Narrow sense heritability $\left(h^{2}\right)$ estimates were calculated as $0.5 * S_{\mathrm{B}}^{2} /\left(0.5 * S_{\mathrm{B}}^{2}+S_{\mathrm{w}}{ }^{2}\right.$ ) (Hegmann \& Possidente, 1981), where $S_{\mathrm{W}}^{2}$ is the variance within strains and $S_{\mathrm{B}}^{2}$ is the variance between strains. $S_{\mathrm{B}}^{2}$ was calculated as $\left(M S_{\mathrm{B}}-M S_{\mathrm{W}}\right) / k$, where $M S_{\mathrm{W}}$ is the mean square within strains, $M S_{\mathrm{B}}$ is the mean squares between strains, and $k$ is an adjusted value for the number of worms per strain (Sokal \& Rohlf, 1969). The sampling variance of $h^{2}$ was calculated as

$$
\left.\left\{2 *[1+(k-1) t]^{2} *(1-t)\right\} /[k *(k-1)] *(N-1)\right]
$$

(Falconer, 1981), where $t$ is the correlation and $N$ is the number of strains. $t$ was calculated as $S_{\mathrm{B}}^{2} /\left[S_{\mathrm{B}}^{2}+S_{\mathrm{W}}^{2}\right]$. The standard error for $h^{2}$ was obtained by taking the square root of its sampling variance.

Estimates of the number of genes controlling the trait $\left(N_{\mathrm{e}}\right)$ were calculated following Taylor (1976) as $R^{2} / 4(2-F) V_{\mathrm{G}}$, where $R$ is the difference between the highest and lowest mean value, $V_{\mathrm{G}}$ is the variance of means of RI strains, and $F$ is the inbreeding coefficient, effectively 1 for these $C$. elegans RI strains.

\section{Strain distribution patterns}

A strain distribution pattern (SDP) is a list that describes which parental alleles are present in each RI strain for a given locus. Three single-gene traits known to be segregating within the RI strains were monitored to construct SDPs for each: a temperature-sensitive (Ts) embryonic-lethal trait (Wood et al., 1980) and a sodium-dodecyl-sulphate (SDS)-sensitive dauer larvae trait (D. L. Riddle, personal communication), both present in BergBO, and a restriction fragment length polymorphism (RFLP) resulting from a $\mathrm{Tc} 1$ insertion into BergBO giving an 8.7-kb fragment in $\mathrm{BergBO}$ and a 7.0-kb fragment in N2 (Emmons \& Yesner, 1984; Johnson, 1986).

The Ts trait was monitored by rearing eggs to adulthood at $25^{\circ} \mathrm{C}$, the temperature at which Ts strains fail to reproduce. SDS resistance was monitored following the protocol of Cassada \& Russell (1975), except that distilled $\mathrm{H}_{2} \mathrm{O}$ was used in all washes. RFLPs were analysed using standard techniques (Maniatis et al., 1982; Emmons \& Yesner, 1984; Johnson, 1986; Simpson et al., 1986).

\section{Results}

Table 1 summarizes the mean life spans of the parental and RI strains in the three trials. The parental midpoint for the life span in each trial was 21.2 days, 20.7 days and 17.3 days, respectively.

TJ142 and TJ143 are the longest-lived RI strains with average life span extensions relative to the parental of 31 and $26 \%$, respectively. The life span of TJ142 was extended to 58 and $37 \%$ in trials 2 and 3 , and the mean life span of TJ143 was extended to 42 and $50 \%$ in trials 2 and 3, relative to the parental midpoints. TJ130 is consistently among the shortest-lived strains. The mean life span of the TJ130 line is reduced by 23 and $20 \%$ in trials 2 and 3, respectively. Trial 1 is anomalous for these and other life spans.

Results for self-fertility from the same individually cultured hermaphrodites for which life span was measured are shown in Table 2 . Self-fertilities in trial 2 were consistently higher than in the other two trials. Values in trial 3 may have been depressed by an elevation in incubator temperature. Self-fertility tends to be lower in liquid culture than in agar culture, which perhaps reflects a lower food concentration or lower oxygen availability in liquid (A. E. Brooks and T. E. Johnson unpublished observations).

A two-way ANOVA (Table 3 ) for life span shows that neither strain nor trial was significant at the $P<0.05$ level; but for self-fertility both trial and strain are highly significant $(P<0.001)$ factors. This two-way ANOVA also confirms that there is a significant $(P<0.001)$ interaction between trial and strain effects for both span and total self-fertility. A variation in strain means and altered ranking of strains between 
Table 1 Mean life span*

\begin{tabular}{lllll}
\hline Strain & Trial 1 $(n) \dagger$ & Trial $2(n)$ & Trial 3 $(n)$ & Overall mean \\
\hline N2 & $18.7 \pm 4.3(6)$ & $20.6 \pm 3.4(8)$ & $15.2 \pm 5.4(9)$ & 18.0 \\
BergBO & $23.7 \pm 5.7(10)$ & $20.7 \pm 4.6(11)$ & $19.5 \pm 2.3(8)$ & 21.4 \\
TJ101 & $24.8 \pm 2.9(8)$ & $16.5 \pm 2.8(8)$ & $18.5 \pm 6.2(12)$ & 19.7 \\
TJ104 & $20.9 \pm 7.2(7)$ & $16.4 \pm 6.0(11)$ & $23.2 \pm 7.6(5)$ & 19.2 \\
TJ106 & $23.1 \pm 7.1(7)$ & $21.9 \pm 6.7(11)$ & $17.9 \pm 6.8(8)$ & 21.0 \\
TJ107 & $17.2 \pm 3.3(5)$ & $25.5 \pm 10.1(8)$ & $16.8 \pm 4.4(8)$ & 20.2 \\
TJ119 & $17.6 \pm 5.2(8)$ & $17.3 \pm 3.3(8)$ & $18.4 \pm 7.8(8)$ & 17.7 \\
TJ124 & $15.7 \pm 5.2(7)$ & $17.1 \pm 7.2(10)$ & $24.4 \pm 10.1(11)$ & 19.6 \\
TJ128 & $18.1 \pm 4.7(7)$ & $17.9 \pm 4.7(10)$ & $19.9 \pm 5.4(11)$ & 18.7 \\
TJ130 & $16.8 \pm 3.0(4)$ & $15.9 \pm 6.3(11)$ & $13.9 \pm 10.6(5)$ & 15.5 \\
TJ132 & $18.6 \pm 5.1(7)$ & $20.3 \pm 5.8(11)$ & $21.3 \pm 5.0(9)$ & 20.2 \\
TJ135 & $19.0 \pm 4.0(8)$ & $22.0 \pm 8.2(11)$ & $23.0 \pm 6.4(10)$ & 21.5 \\
TJ138 & $20.8 \pm 10.3(9)$ & $30.0 \pm 2.8(6)$ & $21.0 \pm 5.2(6)$ & 23.5 \\
TJ141 & $21.0 \pm 4.4(6)$ & $17.5 \pm 5.6(10)$ & $17.3 \pm 6.9(6)$ & 18.4 \\
TJ142 & $18.9 \pm 5.2(7)$ & $32.6 \pm 6.9(11)$ & $23.8 \pm 8.0(12)$ & 25.9 \\
TJ143 & $15.1 \pm 6.2(7)$ & $29.4 \pm 7.6(12)$ & $26.0 \pm 7.7(12)$ & 24.9 \\
\hline
\end{tabular}

* Means and standard deviations for worms that died natural deaths.

$\uparrow$ Number of individuals monitored in each trial.

Table 2 Mean hermaphrodite self-fertility*†

\begin{tabular}{lrlrr}
\hline Strain & \multicolumn{1}{l}{ Trial 1 } & \multicolumn{1}{l}{ Trial 2 } & \multicolumn{1}{c}{ Trial 3 } & Overall mean \\
\hline N2 & $94.3 \pm 12.9$ & $238.4 \pm 32.7$ & $167.1 \pm 31.1$ & 172.9 \\
BergBO & $58.7 \pm 47.1$ & $239.8 \pm 50.4$ & $77.6 \pm 32.5$ & 132.6 \\
TJ101 & $49.4 \pm 21.3$ & $119.4 \pm 39.2$ & $22.2 \pm 10.2$ & 57.7 \\
TJ104 & $69.3 \pm 19.3$ & $108.7 \pm 45.0$ & $48.0 \pm 23.8$ & 83.5 \\
TJ106 & $85.7 \pm 14.2$ & $166.9 \pm 16.8$ & $77.9 \pm 35.0$ & 117.7 \\
TJ107 & $105.0 \pm 23.6$ & $169.1 \pm 37.5$ & $66.8 \pm 28.5$ & 114.9 \\
TJ119 & $55.5 \pm 23.3$ & $119.1 \pm 30.1$ & $59.1 \pm 31.5$ & 77.9 \\
TJ124 & $31.9 \pm 23.3$ & $162.6 \pm 20.3$ & $80.3 \pm 23.1$ & 97.6 \\
TJ128 & $88.0 \pm 20.6$ & $211.9 \pm 17.0$ & $61.5 \pm 35.8$ & 121.8 \\
TJ130 & $31.8 \pm 13.7$ & $178.5 \pm 30.5$ & $26.6 \pm 16.0$ & 111.2 \\
TJ132 & $69.7 \pm 28.9$ & $228.4 \pm 39.6$ & $107.8 \pm 30.5$ & 147.1 \\
TJ135 & $114.6 \pm 28.3$ & $248.5 \pm 29.6$ & $152.1 \pm 35.3$ & 178.3 \\
TJ138 & $90.6 \pm 31.1$ & $180.2 \pm 28.3$ & $84.5 \pm 65.1$ & 114.5 \\
TJ141 & $101.8 \pm 34.2$ & $155.1 \pm 34.8$ & $83.5 \pm 68.5$ & 121.0 \\
TJ142 & $115.1 \pm 19.9$ & $241.9 \pm 38.3$ & $156.8 \pm 35.8$ & 178.3 \\
TJ143 & $112.6 \pm 29.9$ & $132.1 \pm 52.8$ & $123.8 \pm 43.1$ & 124.5 \\
\hline
\end{tabular}

*Means and standard deviations for total self-fertilized progeny in liquid culture for worms that died natural deaths.

tSee Table 1 for number of individuals monitored in each trial.

trials (Tables 1 and 2) also suggests the existence of a trial-by-strain interaction.

\section{Heritabilities}

Estimates of heritabilities (Table 4) indicate the presence of a small genetic component for life span and somewhat larger components for self-fertility from day 3 through to day 6 and for total self-fertility. Heritability estimates were significantly different from 0 for age-specific self-fertility from day 3 through to day 6 in trials 2 and 3 . All three trials showed significant heritabilities for total self-fertility with the heritability estimates of $0.30,0.46$ and 0.43 , respectively. 
Table 3 Analysis of variance of life span and self-fertility

\begin{tabular}{lrrrrr}
\hline Source of variation & Sum of squares & d.f. & Mean square & $F$-test & $P$ \\
\cline { 1 - 2 } Life span & & & & & \\
$\quad$ Main effects & 2352.1 & 17 & 138.4 & 3.5 & 0.000 \\
$\quad$ Trial & 253.9 & 2 & 127.0 & 1.0 & 0.376 \\
$\quad$ Strain & 2098.2 & 15 & 139.9 & 1.1 & 0.386 \\
Interactions & & & & & \\
$\quad$ Trial*strain & 3767.4 & 30 & 125.6 & 3.1 & 0.000 \\
$\quad$ Error & 14478.6 & 362 & 40.0 & & \\
$\quad$ Total & 20598.2 & 409 & 50.4 & & \\
& & & & & \\
Self-fertility & & & & & \\
$\quad$ Main effects & 1311926.3 & 17 & 77172.1 & 67.8 & 0.000 \\
$\quad$ Trial & 883405.6 & 2 & 441702.8 & 60.9 & 0.000 \\
$\quad$ Strain & 428520.7 & 15 & 28568.0 & 3.9 & 0.001 \\
Interactions & & & & & \\
$\quad$ Trial*strain & 217566.3 & 30 & 7252.2 & 6.4 & 0.000 \\
$\quad$ Error & 411739.0 & 362 & 1137.4 & & \\
$\quad$ Total & 1723882.8 & 409 & 4214.9 & & \\
\hline
\end{tabular}

Table 4 Narrow-sense heritabilities of life-history traits

\begin{tabular}{llll}
\hline Character & $\begin{array}{l}\text { Trial 1 } \\
h^{2} \pm \text { S.E. }\end{array}$ & $\begin{array}{l}\text { Trial 2 } \\
h^{2} \pm \text { S.E. }\end{array}$ & $\begin{array}{l}\text { Trial 3 } \\
h^{2} \pm \text { S.E. }\end{array}$ \\
\hline Life span & $0.05 \pm 0.08$ & $0.36 \pm 0.11$ & $0.06 \pm 0.08$ \\
& & & \\
Age-specific & & & \\
self-fertility & & & \\
Day 3 & $0.18 \pm 0.11$ & $0.59 \pm 0.08$ & $0.28 \pm 0.11$ \\
Day 4 & $0.14 \pm 0.07$ & $0.39 \pm 0.10$ & $0.44 \pm 0.10$ \\
Day 5 & $0.09 \pm 0.09$ & $0.55 \pm 0.08$ & $0.36 \pm 0.11$ \\
Day 6 & $0.00 \pm 0.06$ & $0.41 \pm 0.10$ & $0.23 \pm 0.11$ \\
Day 7 & $0.04 \pm 0.08$ & $0.09 \pm 0.08$ & $0.15 \pm 0.10$ \\
Day 8 & $0.04 \pm 0.07$ & $0.09 \pm 0.08$ & $0.01 \pm 0.05$ \\
Total self- & & & \\
fertility & $0.30 \pm 0.11$ & $0.46 \pm 0.09$ & $0.43 \pm 0.10$ \\
\hline
\end{tabular}

The heritability estimate for life span was negligible $(0.05$ and 0.06$)$ and non-significant in trials 1 and 3, respectively, but was significant $(0.36)$ in trial 2 .

\section{Correlations among life span and age-specific self- fertility}

While the phenotypic correlations between life span and total self-fertility were positive in all three trials, they were not significant at the 0.05 level (Table 5). The only statistically significant phenotypic correlation observed between life span and age-specific self-fertility, at 4 days of age in trial 3 , was positive, and except for trial 1, most observed genetic correlations between life span and age-specific or total self-fertility were positive also, although none was significant.

Age-specific fertilities tended to be positively correlated with fertilities on flanking days. This pattern was observed for phenotypic correlations, for which many values were highly significant $(P<0.001)$, and for genetic correlations, some of which were also significant. Note, however, that substantial differences were observed in the correlations between daily self-fertilities among trials. Total self-fertility showed consistently positive and often significant phenotypic correlation with age-specific self-fertilities. Of course, these measures are not independent, but the correlations indicate relative stability of self-fertility over the whole period of reproduction. In some cases, genetic correlations between total self-fertility and age-specific selffertility (at 3 and 4 days of age) were quite high, indicating almost complete genetic co-determination of these traits.

\section{Number of loci}

Estimates of the number of independently segregating loci specifying life span and/or self-fertility indicate that two or three loci specify each trait (Table 6). These are minimum estimates because they are based on the assumptions that (i) the observed extreme values represent the most extreme gene combinations possible, (ii) these traits are specified by genes with equal, additive 
Table 5 Correlation among age-specific self-fertility, total self-fertility, and life span

\begin{tabular}{|c|c|c|c|c|c|c|c|c|c|}
\hline \multirow[b]{2}{*}{ Day } & \multirow[b]{2}{*}{ Trial } & \multicolumn{6}{|c|}{ Daily self-fertility } & \multirow[b]{2}{*}{ Total } & \multirow[b]{2}{*}{ Life span } \\
\hline & & 3 & 4 & 5 & 6 & 7 & 8 & & \\
\hline 3 & 1 & 1 & $0.456^{* * *}$ & 0.187 & 0.134 & -0.051 & -0.087 & $0.825^{* * *}$ & 0.219 \\
\hline 3 & 2 & 1 & $0.410^{* * *}$ & $-0.500^{* * *}$ & $-0.435^{* * *}$ & $-0.272^{*}$ & -0.178 & $0.474^{* * *}$ & 0.082 \\
\hline 3 & 3 & 1 & $0.656^{* * *}$ & $0.361^{* *}$ & 0.071 & -0.003 & -0.122 & $0.877^{* * *}$ & 0.102 \\
\hline 4 & 1 & 0.573 & 1 & $0.398^{* *}$ & 0.278 & 0.151 & 0.244 & $0.573^{* * *}$ & 0.250 \\
\hline 4 & 2 & 0.542 & 1 & 0.182 & 0.108 & 0.039 & -0.015 & $0.845^{* * *}$ & 0.113 \\
\hline 4 & 3 & $0.785^{* *}$ & 1 & $0.676^{* * *}$ & $0.445^{* * *}$ & $0.286^{*}$ & 0.043 & $0.840^{* * *}$ & $0.304^{*}$ \\
\hline 5 & 1 & 0.056 & 0.386 & 1 & $0.415^{* * *}$ & $0.337^{*}$ & 0.145 & $0.437^{* * *}$ & 0.003 \\
\hline 5 & 2 & -0.637 & 0.139 & 1 & $0.744^{* * *}$ & $0.403^{* * *}$ & $0.303^{* *}$ & $0.388^{* * *}$ & 0.056 \\
\hline 5 & 3 & 0.611 & $0.852^{* *}$ & 1 & $0.562^{* * *}$ & $0.354^{* *}$ & 0.114 & $0.599^{* * *}$ & 0.267 \\
\hline 6 & 1 & 0.500 & 0.331 & -0.096 & 1 & $0.354^{* *}$ & 0.118 & $0.406^{* * *}$ & 0.056 \\
\hline 6 & 2 & -0.622 & 0.122 & $0.963^{* * *}$ & 1 & $0.676^{* * *}$ & $0.286^{*}$ & $0.377^{* * *}$ & 0.086 \\
\hline 6 & 3 & 0.066 & 0.469 & $0.769^{*}$ & 1 & $0.726^{* * *}$ & $0.294^{*}$ & $0.340^{* *}$ & 0.128 \\
\hline 7 & 1 & 0.224 & 0.668 & 0.428 & 0.504 & 1 & $0.521^{* * *}$ & 0.117 & -0.116 \\
\hline 7 & 2 & -0.649 & 0.037 & $0.906^{* * *}$ & $0.946^{* * *}$ & 1 & $0.361^{* * * *}$ & 0.245 & 0.032 \\
\hline 7 & 3 & -0.131 & 0.268 & 0.599 & $0.966^{* * *}$ & 1 & $0.431^{* * *}$ & 0.237 & -0.000 \\
\hline 8 & 1 & 0.064 & 0.506 & 0.137 & 0.288 & 0.716 & 1 & 0.040 & -0.057 \\
\hline 8 & 2 & -0.482 & -0.020 & $0.717^{*}$ & $0.760^{*}$ & 0.691 & 1 & 0.112 & 0.016 \\
\hline 8 & 3 & -0.189 & 0.204 & 0.495 & $0.775^{*}$ & $0.818^{* *}$ & 1 & 0.032 & 0.056 \\
\hline \multicolumn{10}{|l|}{ Total } \\
\hline 1 & & $0.934^{* * *}$ & 0.598 & 0.237 & 0.562 & 0.372 & 0.241 & 1 & 0.110 \\
\hline 2 & & 0.484 & $0.908^{* * *}$ & 0.303 & 0.341 & 0.247 & 0.176 & 1 & 0.174 \\
\hline 3 & & $0.903^{* * *}$ & $0.902^{* * *}$ & $0.803^{* *}$ & 0.366 & 0.182 & 0.113 & 1 & 0.143 \\
\hline \multicolumn{10}{|c|}{ Life span } \\
\hline 1 & & 0.182 & 0.217 & -0.345 & -0.046 & -0.175 & -0.206 & -0.076 & 1 \\
\hline 2 & & 0.142 & 0.263 & 0.069 & 0.048 & 0.081 & -0.007 & 0.296 & 1 \\
\hline 3 & & 0.276 & 0.625 & 0.439 & 0.320 & 0.193 & 0.192 & 0.344 & 1 \\
\hline
\end{tabular}

Phenotypic correlations above diagonal; genetic correlations below. Significance: ${ }^{*} P<0.05 ;{ }^{* *} P<0.01 ;{ }^{* * *} P<0.001$;

Bonferroni test. A preliminary version of this table appeared in Johnson et al. (1990).

Table 6 Estimate* of number of loci $\left(N_{\mathrm{e}}\right)$ influencing life span and/or self-fertility

\begin{tabular}{lrrr}
\hline & Trial 1 & Trial 2 & Trial 3 \\
\hline Lifespan & & & \\
$R$ & 9.6 & 16.7 & 12.1 \\
$V_{\mathrm{G}}$ & 7.8 & 28.5 & 11.8 \\
$N_{\mathrm{e}}$ & 2.9 & 2.4 & 3.1 \\
Self-fertility & & & \\
$R$ & 83.4 & 139.8 & 144.9 \\
$V_{\mathrm{G}}$ & 791.1 & 2321.3 & 1916.7 \\
$N_{\mathrm{e}}$ & 2.2 & 2.1 & 2.7 \\
\hline
\end{tabular}

*See text for formula and explanation of terms.

effects, and (iii) the genes specifying these traits segregate independently (Taylor, 1976). These assumptions impose dramatic upper limits on the gene number estimates (Lande, 1981) and will be discussed later.

\section{Genetic mapping of QTLs}

Table 7 shows the strain distribution pattern (SDP) for three single-gene differences which segregate within these RI strains: a Bergerac gene mapped to linkage group II (Wood et al., 1980), which results in a temperature-sensitive embryogensis phenotype, a Bergerac gene causing SDS-sensitive dauers (D. L. Riddle, personal communication), and a restriction fragment length polymorphism (RFLP) on chromosome 5 (Emmons \& Yesner, 1984). The data presented in Table 7 show that all three single-gene markers segregate independently. Recombination frequencies of approximately $50 \%$ among the three markers were observed.

The linkage of QTLs (Lander \& Botstein, 1989) that specify length of life or hermaphrodite self-fertility to these three single genes was tested as follows. For each single-gene trait, all 16 strains were partitioned by allelic type into groups. If life span or fertility is linked 
Table 7 Strain distribution

\begin{tabular}{lccc}
\hline Genotype & Ts* & $\begin{array}{l}\text { SDS } \\
\text { resistancet }\end{array}$ & $\begin{array}{l}\text { RFLP+ } \\
(\mathbf{k b})\end{array}$ \\
\hline N2 & + & + & 7.0 \\
BergBO & - & - & 8.7 \\
TJ101 & - & + & 8.7 \\
TJ104 & - & + & 8.7 \\
TJ106 & - & - & - \\
TJ107 & - & - & 7.0 \\
TJ119 & - & + & 7.0 \\
TJ124 & \pm & + & 8.7 \\
TJ128 & - & + & 8.7 \\
TJ130 & - & + & 7.0 \\
TJ132 & + & + & 7.0 \\
TJ135 & + & - & 8.7 \\
TJ138 & + & + & 7.0 \\
TJ141 & + & + & - \\
TJ142 & + & - & 8.7 \\
TJ143 & + & + & 7.0 \\
\hline
\end{tabular}

*Temperature sensitivity: +strains reproduce at $25^{\circ} \mathrm{C}$;

- strains do not.

†Strains form SDS-resistant dauer larvae; - strains do not.

$\ddagger$ Restriction fragment length polymorphism: used pCE1001

which maps to linkage group V (Emmons \& Yesner, 1984;

Johnson, 1986).

to one of the three traits, there will be a significant difference between the groups partitioned by alleles of that trait. Survival comparisons for life span and $t$-test for fertility were made between each grouping of strains by allele (Table 8 ). The scoring of the traits in some strains was ambiguous, so the data were initially analysed with the ambiguous strains excluded. The data were also analysed by including the ambiguous strains in both classes and in no case did inclusion of these equivocal strains significantly affect the results.

A QTL for fertility was significantly associated with both the temperature-sensitive and the SDS-resistant dauer trait in all three assays. Life span was significantly associated with these same two traits 2 but not in trials 1 or 3 . The locus detected by the RFLP was not significantly associated with either trait in any trial.

\section{Discussion}

We have addressed several issues concerning the genetic specification of senescence and hermaphrodite fertility in C. elegans. While the number of strains in our experiments is small, there are, nevertheless, notable trends that should be studied further.
Table 8 Life span and hermaphrodite self-fertility of groups partitioned by strain distribution patterns

\begin{tabular}{|c|c|c|c|}
\hline \multirow{2}{*}{$\begin{array}{l}\text { Trial } \\
\text { and } \\
\text { character }\end{array}$} & \multicolumn{3}{|c|}{ Probability of significance* } \\
\hline & Ts $\dagger$ & $\begin{array}{l}\text { SDS } \\
\text { resistancef }\end{array}$ & RFLPS \\
\hline \multicolumn{4}{|l|}{ Trial no. 1} \\
\hline Life span & 0.17 & 0.35 & 0.12 \\
\hline Fertility & $<0.001$ & 0.007 & 0.36 \\
\hline \multicolumn{4}{|l|}{ Trial no. 2} \\
\hline Life span & $<0.001$ & $<0.001$ & 0.24 \\
\hline Fertility & $<0.001$ & $<0.001$ & 0.13 \\
\hline \multicolumn{4}{|l|}{ Trial no. 3} \\
\hline Life span & 0.05 & 0.90 & 0.19 \\
\hline Fertility & $<0.001$ & 0.001 & 0.32 \\
\hline $\begin{array}{l}\text { *Life span: } I \\
\text { fertility: } P \text {-v } \\
\text { †RI strain TJ } \\
\neq \text { RI strains T } \\
\text { assay and ex } \\
\text { \&RI strains }\end{array}$ & $\begin{array}{l}\text { rom SY } \\
\text { n } t \text {-test } \mathrm{t} \\
\text { cluded d } \\
\text { a } \mathrm{TJ} 143 \\
\text { a } \mathrm{TJ} 14\end{array}$ & $\begin{array}{l}\text { survival proc } \\
\text { en means (two } \\
\text { ambiguous Ts } \\
\text { ambiguous } i \\
\text { excluded fro }\end{array}$ & $\begin{array}{l}\text { d). } \\
\text { y results. } \\
\text { SDS } \\
\text { alysis. }\end{array}$ \\
\hline
\end{tabular}

\section{Heritabilities}

Our estimates of heritability of life span were low in comparison to the $19-51 \%$ heritabilities obtained for life span in an earlier study (Johnson \& Wood, 1982). These lower estimates may be due to the more extensive manipulation required by individual nematode cultures.

Estimates of $30-46 \%$ of the total self-fertility are due to the additive genetic effects in this genetic background (Table 4). These estimates are high for a trait so closely associated with fitness. These RI strains were generated in a way that minimized selection for both fertility and life span, but some selection certainly has occurred. Our results, however, corroborate other studies. For example, Rose \& Charlesworth (1981) and Service \& Rose (1985) found high additive genetic variance for fecundity (number of eggs laid) measured during 24-h periods in a laboratory sample of young female fruit flies. In addition, significant variability was observed in populations of Drosophila used in direct selection of longer reproduction times (Luckinbill et al., 1984; Rose, 1984a).

\section{Number of loci}

This analysis suggests that life span and self-fertility is each specified by a minimum of two to three genes. Again, this estimate is probably a substantial under- 
estimate because it is constrained in several ways. First, only genes that differ between the parental strains are measured; thus only those genes that differ between the BergBO and the N2 strains are included in the estimate. Second, the method used to estimate the number of genes is itself constrained by the three assumptions mentioned earlier (i.e. that the highest and lowest possible mean values are represented among the genotypes used in the analysis, that all loci have equal, additive effects and no epistasis, and that the loci of interest are unlinked). The first two assumptions are probably not met, and the third is certainly not. As the entire genetic map of $C$. elegans is only about 300 map units and most genes are clustered in the center of six linkage groups (Swanson et al., 1984), the number of independently segregating loci is limited to slightly more than six. These estimates of two to three loci specifying life span or fertility agree with previously published estimates for C. elegans (Johnson, 1986).

\section{Correlations among the total and age-specific fertilities and life span}

Antagonistic pleiotropy between reproduction and life span, a theory originally formulated by Medawar (1946, 1952), has received prominent attention in many models for the evolution of senescence (Charlesworth, 1980; Rose, 1985). This theory suggests that senescence and limited species-specific life spans result from indirect effects on the life span of genes whose primary function involves an increase in overall fitness. In contrast to the prediction of such models of negative correlations, we observed mostly positive genetic correlations between life span and hermaphrodite daily self-fertility. Positive phenotypic correlations between life span and daily self-fertility were observed through day 6 and were also seen between life span and total self-fertility. Similar positive genetic correlations were observed in inbred Drosophila strains by Rose (1984b) who suggested that this correlation arose from inbreeding depression and is therefore spurious. As C. elegans does not show inbreeding depression (Johnson \& Wood, 1982; T. E. Johnson, in preparation), we think that this explanation is unlikely $m$ this instance.

Phenotypic correlations between age-specific hermaphrodite fertility at different ages were positive and significant when fertilities on adjacent days were assayed but tended to show negative pleiotropy between early and late fertility. Negative phenotypic correlations were almost restricted to comparisons between early (day 3) and late (days 7 and 8) reproduction. Such negative correlations between early and late reproduction might be explained, in part, by the fact that sperm number limits hermaphrodite self-fertility (Ward \& Carrel, 1979).

Genetic correlations were almost always positive when adjacent days were assayed. All significant genetic correlations were positive and most of these were for adjacent days. Some evidence of negative pleiotropy was seen but was not statistically significant.

\section{Environmental effects}

Similar heritabilities of total self-fertility were observed in each experiment even though variations in mean values were observed among trials, as indicated by the significant trial effects on self-fertility. Known differences between experiments (e.g. uncontrolled fluctuation of incubator temperature in trial 3, seasonal laboratory temperature variations, and slight differences in procedures) can account for some of the observed variation in mean scores. In addition, some variation may be due to differences among batches of $E$. coli that are used as the food source and to differences in technical skills between investigators.

\section{Conclusion}

There have been few attempts to measure directly the number of loci specifying life span and/or fertility in wild populations. The notion that many (even most) genes are involved in specifying aging has been fostered by the fact that most induced mutations shorten life span (Lints, 1978). However, work on such induced mutations cannot realistically be used to address questions about the evolution of life-history traits because the non-specific general loss of fitness associated with such mutations confounds results and represents alleles not, or only rarely, found in nature.

The extent to which our observations reflect the results of evolution in $C$. elegans is necessarily limited by the artificial environment and the use of laboratory strains. The parental strains used in this study have been maintained in the laboratory (interrupted by periods of freezing) for about 40 years. These populations have not been under selection for length of life but some inadvertent selection for self-fertility has probably occurred during periods of laboratory growth.

Again, in contrast to other organisms, inbreeding depression is not observed in C. elegans (Johnson \& Wood, 1982), perhaps because it is a self-fertilizing hermaphroditic species and therefore normally inbred. This means that the positive correlations between life span and fertility cannot be simply explained by inbreeding depression. Because RI strains contain gene combinations not found in either parent, the genes 
might not operate together as would the parental combinations. If such co-evolved sets of loci are the rule, we would expect outbreeding depression to occur in C. elegans. This has not been observed (Johnson \& Wood, 1982; T. E. Johnson, in preparation). We did find some RI strains, however, with both short life span and low self-fertility. In fact, TJ130 was notable for its reduced overall vigour, which could indicate suboptimal function of new gene combinations for a trait that influences both longevity and self-fertility (e.g. food assimilation). Further studies will entail larger numbers of RI strains and the use of multiple linked RFLPs to localize QTLs (Paterson et al., 1988; Lander \& Botstein, 1989) that specify life span and/or agespecific fertility.

\section{Acknowledgements}

We would like to thank N. L. Foltz, P. Cuccaro and M. Keller for help in data collection and W. L. Conley, H. Caravantes and Y. Davoodbhoy for other technical help. We thank N. L. Foltz and R. Davis for useful discussions, and we acknowledge J. C. DeFries for first suggesting the use of RI strains to us and D. W. Fulker for providing formulae for the estimation of error terms in heritability. This research is supported by research grants from the National Institute on Aging (AG05720 and AG08322), the National Science Foundation (8208652), the American Federation for Aging Research, and by a Research Career Development Award to TEJ (AG00369). Some stocks were supplied by and are available through the Caenorhabditis Genetics Center, which is supported by contract N01-AG-9-2113 between the National Institutes of Health and the curators of the University of Missouri.

\section{References}

BAILEY, D. W. 1971. Recombinant-inbred strains, an aid to finding identity, linkage, and function of histocompatibility and other genes. Transplantation, 11, 325-327.

BLIZARD, D. A. AND BAILEY, D. w. 1979. Genetic correlation between open-field activity and defecation: Analysis with the CXB recombinant-inbred strains. Behav. Genet., 9, 349-357.

BRENNER, S. 1974. The genetics of Caenorhabditis elegans. Genetics, 77, 71-94.

CASSADA, R. AND RUSSELL, R. L. 1975. The dauerlarva, a postembryonic development variant of the nematode Caenorhabditis elegans. Dev. Biol., 46, 326-342

CHARLESWORTH, B. 1980. Evolution in Age-Structured Populations. Cambridge University Press, Cambridge, England.

EMMONS, S. W., KLASS, M. R. AND HIRSH, D. 1979. Analysis of the constancy of DNA sequences during development and evolution of the nematode Caenorhabditis elegans. Proc. Natl. Acad. Sci., USA, 76, 1333-1337

EMMONS, S. W. AND YESNER, L. 1984. High-frequency excision of transposable element $\mathrm{Tc} 1$ is limited to somatic cells. Cell, 36, 599-605

FALCONER, D. S. 1981. Introduction to Quantitative Genetics. Longman, New York, NY, USA.

FRIEDMAN, D. B. AND JOHNSON, T. E. 1988. A mutation in the age1 gene in Caenorhabditis elegans lengthens life and reduces hermaphrodite fertility. Genetics, 118, 75-86.

HEGMANN, J. P. AND possidente, B. 1981. Estimating genetic correlations from inbred strains. Behav. Genet., 11, 103-114.

JOHNSON. T. E. 1986. Molecular and genetic analysis of a multivariate system specifying behavior and life span. Behav. Genet., 16, 221-235.

JoHnson, T. E. AND wood, w. B. 1982. Genetic analysis of lifespan in Caenorhabditis elegans. Proc. Natl. Acad. Sci., USA, 79, 6603-6607.

LANDE, R. 1981. The minimum number of genes contributing to quantitative variation between and within populations. Genetics, 99, 541-543.

LANDE, R. 1982. A quantitative genetic theory of life-history evolution. Ecology, 63, 607-615.

LANDER, E. S. AND BOTTSTEIN, D. 1989. Mapping Mendelian factors underlying quantitative traits using RFLP linkage maps. Genetics, 121, 185-199.

LINTS, F. A. 1978. Genetics and Ageing. Karger, Basel.

LUCKINBILL, L. S., ARKING, R., ClARE, M. J., CIROCCO, W. S. AND BUCK, S. A. 1984. Selection for delayed senescence in Drosophila melanogaster. Evolution, 38, 996-1003.

MANIATIS, T., FRITSCH E. F. AND SAMBROOK, J. 1982. Molecular Cloning: A Laboratory Manual. Cold Spring Harbor Laboratory, Cold Spring Harbor, New York, NY, USA.

MEDAWAR, P. B. 1946. Old age and natural death. Modern Quart., 1, 30-56.

Medawar, p. B. 1952. An Unsolved Problem of Biology. H. K. Lewis, London, England.

NICHOlas, W. L., DOUGHERTY, E. C. AND haNSEN, E. L. 1959. Axenic cultivation of Caenorhabditis briggsae (Nematoda: Rhabditidae) with chemically undefined supplements: comparative studies with related nematodes. Ann. N.Y. Acad. Sci., 77, 218-238.

NIGON, v. 1949. Les modalités de la reproduction et le determinisme de sexe chez quelque nematodes libres. Ann. Nat. Zool. Ser., 11, 1-132.

PATERSON, A. H., LANDER, E. S., HEWTTT, J. D., PETERSON, S., LINCOLN, S. E. AND TANKSLEY, S. D. 1988. Resolution of quantitative traits into Mendelian factors by using a complete linkage map of restriction fragment length polymorphisms. Nature, 335, 721-726.

ROSE, M. R. 1984a. Laboratory evolution of postponed senescence in Drosophila melanogaster. Evolution, 38, 1004-1010.

ROSE, M. R. 1984b. Genetic covariation in Drosophila life history: untangling the data. Am. Nat., 123, 565-569.

ROSE, M. R. 1985. Life history evolution with antagonistic pleiotropy and overlapping generations. Theor. Pop. Biol., 28, 342-358. 
ROSE, M. R. AND CHARLESWORTH, B. 1981. Genetics of life history in Drosophila melanogaster. II. Exploratory selection experiments. Genetics, 97, 187-196.

ROSE, M. R., SERVICE, P. AND HUTCHINSON, E. W. 1987. Three approaches to trade-offs in life-history evolution. In: Loesccke, V. (ed.) Genetic Constraints on Adaptive Evolution, Springer-Verlag, Berlin, Germany.

SERVICE, P. M. AND ROSE, M. R. 1985. Genetic covariation among life-history components: the effect of novel environments. Evolution, 39, 943-945.

SIMPSON, V. J., JOHNSON, T. E. AND HAMMEN, R. F. 1986. Caenorhabditis elegans DNA does not contain 5methylcytosine at any time during development or aging. Nucl. Acids Res., 14, 6711-6719.

SOKAL, R. R. AND ROHLF, F. J. 1969. Biometry. W. H. Freeman and Company, San Francisco, CA, USA.

STEINBERG, D. AND COLLA, P. 1988. SURVIVAL: $A$ supplementary module for SYSTAT. SYSTAT, Inc., Evanston, IL, USA.
SWANSON, M. M., EDGLEY M. L. AND RIDDLE, D. L. 1984. The nematode Caenorhabditis elegans. Genet. Maps, 3, 286-299.

TAYLOR, B. A. 1976. Genetic analysis of susceptibility to isoniazid-induced seizures in mice. Genetics, 83, 373-377.

WARD, S. AND CARREL, J. S. 1979. Fertilization and sperm competition in the nematode Caenorhabditis elegans. Dev. Biol., 73, 304-321.

WILKINSON, L. 1984. SYSTAT: The System for Statistics. SYSTAT, Inc., Evanston, IL, USA.

WILliaMs, G. C. 1957. Pleiotropy, nature selection, and the evolution of senescence. Evolution, 11, 398-411.

wood, w. B. (ED.) 1988. The Nematode: Caenorhabditis elegans. Cold Spring Harbor Laboratory, Cold Spring Harbor, New York, NY, USA.

WOOD, W. B., HECHT, R., CARR, S., VANDERSLICE, R., WOLF, N. AND HIRSH, D. 1980. Parental effects and phenotypic characterization of mutations that affect early development in Caenorhabditis elegans. Dev. Biol., 74, 446-469. 